\title{
Full complex Fresnel holograms displayed on liquid crystal devices
}

\author{
R. Tudela, E. Martín-Badosa, I. Labastida, E. Pleguezuelos, S. Vallmitjana, \\ I. Juvells, and A. Carnicer \\ Universitat de Barcelona. Laboratori d’Òptica. Dept. Física Aplicada i Òptica. \\ Avda. Diagonal 647.08028 Barcelona. Spain.raul@optica.fao.ub.es
}

\begin{abstract}
We propose an implementation to display full complex information using two ferroelectric liquid crystals spatial light modulators. We compare the simulated and experimental results obtained.
\end{abstract}

Keywords: full complex modulation, liquid crystal devices, Fresnel holography.

\section{INTRODUCTION}

Many optical applications require an accurate display of full complex information, which involves the use of devices able to control amplitude and phase. Last years the use of liquid crystal devices (LCD) has become a common way to display complex information, even though their modulation capabilities are limited. In an ideal case, LCDs able to simultaneously modulate amplitude and phase in a wide range of values would be required. At the present time such devices are not available, therefore it is necessary to choose a strategy to represent the complex plane: to use codifying methods with a single panel ${ }^{1}$, which implies a loss of resolution, or to use at least two panels, which entails the design of a more complex optical system ${ }^{2,3}$. Nowadays there are devices able to work in real-only and imaginary-only configurations ${ }^{4}$, thereby it is possible to represent the complex unity plane by using two of these panels in an optical system in which the real and imaginary parts are added.

In this work we present an implementation of this additive method to represent Fresnel holograms. The idea is to compute a digital hologram in order to recover an object at a certain distance from the plane where the hologram will be displayed. The hologram is obtained by retropropagating the information of an object using the inverse Fresnel transform to a distance $-\mathrm{d}$ as follows.

Let $f(\mathrm{x}, \mathrm{y})$ be the original object to be codified in the hologram. Its Fresnel retropropagation to a distance $-d$ is:

$$
U(x, y,-d)=\frac{e^{-i k d}}{-i \lambda d} \int_{-\infty}^{\infty} f\left(x^{\prime}, y^{\prime}\right) \quad \exp \left[-\frac{i k}{2 d}\left(\left(x-x^{\prime}\right)^{2}+\left(y-y^{\prime}\right)^{2}\right)\right] d x^{\prime} d y^{\prime}
$$

where $\mathrm{k}=2 \pi / \lambda$ is the wave number, and $(\mathrm{x}, \mathrm{y})$ and $\left(\mathrm{x}^{\prime}, \mathrm{y}^{\prime}\right)$ are the transversal coordinates in the planes $z=-d$ and $z=0$, respectively.

The information to be displayed, $U(\mathrm{x}, \mathrm{y},-d)$, is a complex function that can be represented as an addition of its real and imaginary parts:

$$
U(x, y,-d)=\operatorname{Re}[U(x, y,-d)]+\operatorname{Im}[U(x, y,-d)]
$$

These two parts are separately displayed on two different panels, one in real-only configuration and the other one in imaginary-only configuration. By propagating and adding these two wavefronts we recover the original object at a distance $d$.

\section{SIMULATED RESULTS}

Before using the optical system, we have performed simulations using the operating modes of our real panels. These panels are reflective ferroelectric liquid crystals spatial light modulators (FLC-SLM) from BNS $^{4}$ with $128 \times 128$ pixels and a pixel pitch of $40 \mu \mathrm{m}$. The light polarization conditions determine the modulation behaviour of the SLM, i.e., the complex values that the panel can represent. Among other configurations, these FLCs can operate in real-only or imaginary-only modes, thus allowing the representation of any complex value by adding its real and imaginary parts displayed on two different devices.

The simulation process starts by fitting the real and imaginary values obtained from the retropropagated information into the real-only and imaginary-only operating modes, respectively. Then, the adjusted values are propagated to the desired plane, where the two parts are added and the reconstruction of the image is achieved. The same process is carried out using the information of just one of the panels to see the differences between reconstructions obtained only from the real or the imaginary part. 
Figure 1 shows the original image to reconstruct and Figure 2 the simulated reconstructions using (a) the full complex information, (b) only the real information and (c) only the imaginary information at a distance of $324 \mathrm{~mm}$.

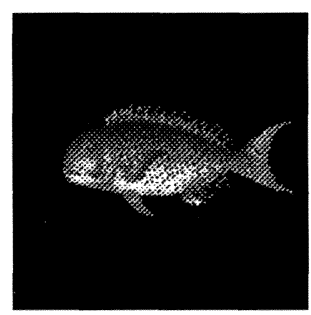

Figure 1. Original object

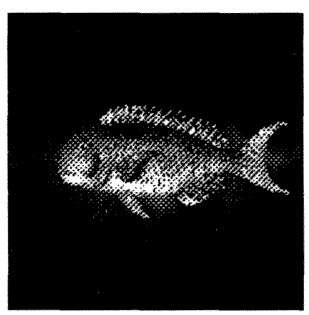

Figure 2. Simulated reconstructions: (a) full complex, (b) real only and (c) imaginary only at $324 \mathrm{~mm}$
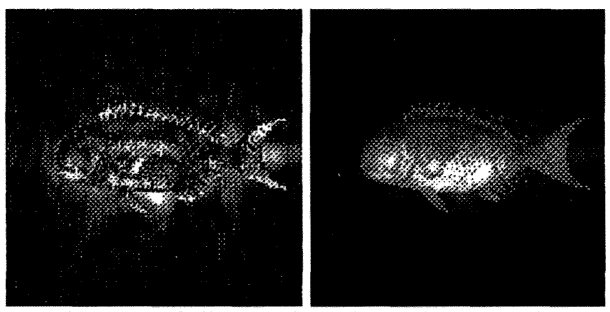

\section{EXPERIMENTAL RESULTS}

We have also carried out some optical experiments using the aforementioned panels. Among the different optical architectures that allow the addition of two wavefronts, we have chosen the one pictured in Figure 3 . The setup consists of two panels arranged in an interferometric configuration, which uses a beam splitter to achieve the addition of the real and the imaginary information displayed on each of the panels. The choice of this configuration derives from the reflective operating mode of the FLC-SLMs used. The main difficulty of this setup is the alignment of the panels to perform the correct pixel to pixel correspondence.

Figure 4 shows the experimental reconstructions using (a) the full complex information, (b) only the real information and (c) only the imaginary information at a distance of $324 \mathrm{~mm}$.

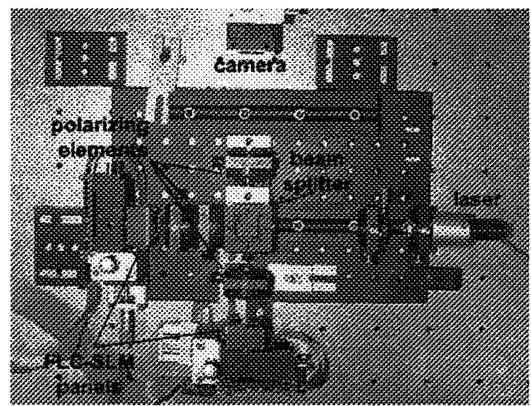

Figure 3. Optical setup

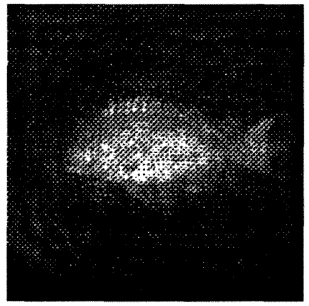

Figure 4. Experimental reconstructions (a) full complex, (b) real only and (c) imaginary only at $324 \mathrm{~mm}$
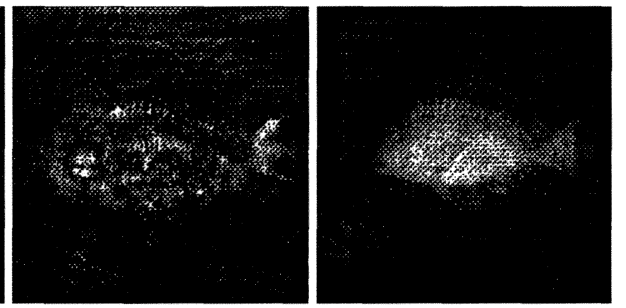

4. CONCLUSIONS

We have proposed a method to display Fresnel holograms using two FLC-SLMs. These devices can operate in a realonly or imaginary-only configurations, and thus the combination of two of them allows to display any complex value of the unity plane. The simulation performed and the optical reconstructions show a reasonable agreement taking into account the limitations of the devices used.

\section{ACKNOWLEDGEMENTS}

This work has been partially funded by the Spanish CICYT through project DPI 2001-3365.

\section{REFERENCES}

1. P. Birch, R. Young, C. Chatwin, M. Farsari, D. Budgett, and J. Richardson, "Fully complex optical modulation with an analogue ferroelectric liquid crystal spatial light modulator", Opt. Commun. 175, pp. 347-352, 2000.

2. R.D. Juday, and J.M. Florence, "Full complex modulation with one-parameter SLMs", Proc. SPIE 1558, pp. 499-504, 1991.

3. D.A. Gregory, J.C. Kirsch, and E.C. Tam, "Full complex modulation using liquid-crystal televisions" Appl. Opt. 31, pp. 163-165, 1992.

4. S.A. Serati, G.D. Sharp, R.A. Serati, D.J. McKnight, J.E. Stockley, "128x128 analog liquid crystal spatial light modulator" Proc. SPIE 2490, pp. 378-387, 1995. 